\title{
Studiengang
}

Ivo Macek*

\section{Weiterbildung am SII - Eine fast dreißigjährige Erfolgsgeschichte}

https://doi.org/10.1515/iwp-2020-2111

Die Informationswissenschaft hat an der Fachhochschule Graubünden in Chur eine lange Tradition. Was viele jedoch nicht wissen: Der Ursprung des Fachbereichs geht auf ein im Jahre 1992 an der Hochschule für Technik und Architektur HTA (so hieß die FH Graubünden damals) erstmals durchgeführtes „Nachdiplomstudium in Information und Dokumentation“ (NDS IuD) zurück, das bemerkenswerterweise auf Initiative eines Dokumentars des in der Nähe von Chur ansässigen Chemie-Konzerns „EMS Chemie“ zustande kam. Erst sechs Jahre später, im Jahre 1998, wurde dann ein Fachhochschuldiplomstudium aus der Taufe gehoben, das seit der Bologna-Reform nun als Bachelor of Science in Informationswissenschaft in Erscheinung tritt. So gesehen ist ein aus Dokumentars-Kreisen initiiertes Weiterbildungsstudium „schuld“, dass sich in Chur die Informationswissenschaft ansiedeln und entwickeln konnte. Heute bildet das Schweizerische Institut für Informationswissenschaft, gemessen am Umsatz und dem Headcount, den größten Fachbereich an der FH Graubünden.

\section{Vom NDS zum MAS}

Doch zurück zum NDS IuD: Seit seiner Lancierung entpuppt sich der Studiengang als echter „Dauerbrenner“ und als konstantestes Weiterbildungsangebot an der Bündner Fachhochschule: Anfänglich im Zweijahresrhythmus gestartet, wird der Kurs seit 1999 jährlich mit einem Klassenzug mit maximal 25 Teilnehmenden durchgeführt. Die eingangs erwähnte Bologna-Reform verwandelte dann das NDS IuD in ein Master of Advanced Studies

*Kontaktperson: Prof. Ivo Macek, Fachhochschule Graubünden, Schweizerisches Institut für Informationswissenschaft, Pulvermühlestrasse 57, 7000 Chur, Schweiz, E-Mail: ivo.macek@fhgr.ch in Information Science (MAS IS)1. Geändert haben sich in den bald dreißig Jahren seines Bestehens auch die Inhalte, doch geblieben ist ein zentrales Merkmal, das bis heute Bestand hat: Das Profil. Das damalige NDS IuD wie der heutige MAS IS ist eine Quereinsteigerausbildung und spricht Personen mit abgeschlossenem Hochschulstudium (egal welcher Fachrichtung) an, die in einem kurzen, aber intensiven und kompakten Studium - die Studiendauer beträgt insgesamt eineinhalb Jahre - das Handwerk bibliothekarischen, archivarischen und dokumentarischen Arbeitens erlernen wollen, um in der Bibliotheks-, Archiv-, Dokumentations- oder Museumsbranche beruflich Fuß fassen zu können. Zusammen mit einer fundierten informationswissenschaftlichen Grundbildung erhalten die Studierenden in kompakter Weise die wichtigsten Werkzeuge und Methoden für eine spätere Tätigkeit als Information Professional mit auf den Weg.

\section{Integriertes Studienkonzept als Erfolgsrezept}

Die Besonderheit dieses Studienangebots - und wahrscheinlich auch sein Erfolgsrezept - ist, dass die Arbeitswelten von Bibliotheken, Archiven und Dokumentationsstellen nicht gesondert betrachtet werden. Vielmehr wird den Studierenden eine integrierte Ausbildung angeboten, die darauf zielt, sich grundlegende Kenntnisse der Arbeitsprozesse aller informationsverarbeitenden Organisationen anzueignen: vom Speichern zum Verwahren und Konservieren bis hin zum aktiven Präsentieren und Ver-

1 Der MAS ist als zugelassene Studienform an Fachhochschulen und Universitäten der Schweiz und Österreichs einzigartig, es handelt sich dabei also quasi um eine austro-helvetische Erfindung, die im Zuge der Bologna-Bildungsreform ab 2006/2007 erstmals auftauchte. Der MAS ist ein postgraduales Studienangebot und gehört damit gemäß eidgenössischer Begriffstypologie zu den Abschlüssen der Stufe Weiterbildung, währenddessen Titel wie BSc/BA, MSc/MA zur sog. grundständigen Bildung gehören. 


\section{Studienkonzept ab 2020}

$-$

"Master of Advanced Studies FHO Information Science»

60 ECTS
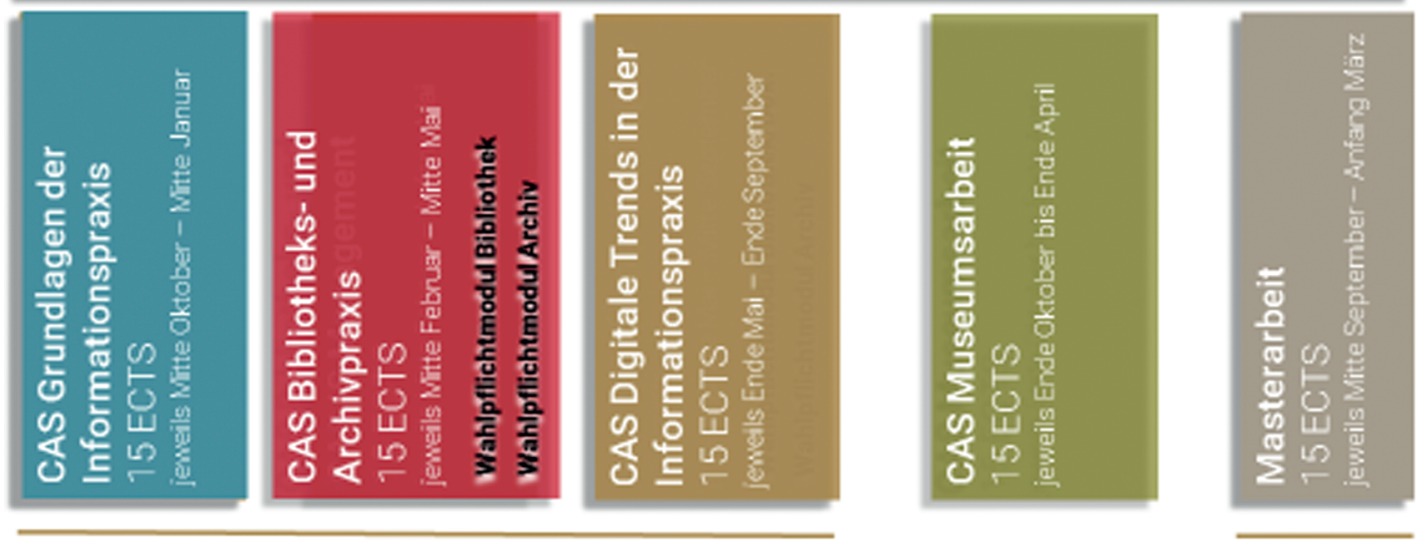

FH

Abbildung 1: Studienkonzept MAS Information Science FH Graubünden (ab Herbst 2020).

mitteln. Denn die IT-gestützte Suche, Darstellung, Auswertung, Verwahrung, Präsentation und Vermittlung von Information ist ein zentrales Element in der Tätigkeit eines Information Professionals.

Beispiel gefällig? Wer in einer Bibliothek mit öffentlichem Sammelauftrag tätig ist, sieht sich zusehends mit Fragen der digitalen Langzeitarchivierung konfrontiert. Ein Thema, das bis dato eher den Archiven vorbehalten war. Dagegen sehen sich staatliche und private Archive zunehmend mit dem Auftrag konfrontiert, ihre Bestände zu vermitteln, gleichsam einem Museum. Archive werden nicht mehr nur von Genealogen, Geschichtsforschern und Studierenden heimgesucht. Wie auch Bibliotheken nicht mehr nur Leseratten anziehen, sondern Menschen allerlei Couleur wie wir in der Schweiz sagen und mit unterschiedlichen Bedürfnissen.

\section{Berufsbegleitend und praxisnah}

Die Formel für den MAS ist simpel: Die Studierenden besuchen drei Zertifikatskurse (neudeutsch CAS) à 15 ECTS und schreiben anschließend eine Masterarbeit à 15 ECTS, was zum mit 60 ECTS dotierten MAS-Abschluss führt. Auf dem Weg dorthin verstreichen rund 18 Monate, in denen die Studierenden 600 Präsenzlektionen besuchen und dabei einen Gesamtarbeitsaufwand von geschätzten 1800 Stunden erbringen.
Der praxisnahe Unterricht wird dabei um Exkursionen ergänzt, die Studierende in die ganze Schweiz führen. Das kommt den meisten entgegen, liegt Chur doch in der Wahrnehmung des Durchschnittsschweizers eher „hinter den sieben Bergen bei den sieben Zwergen“.

\section{Treiber für die jüngste Curriculumsreform}

Wie sieht die Bibliothek der Zukunft aus? Mit welchen Herausforderungen sehen sich Archive konfrontiert? Wie arbeiten Dokumentarinnen und Dokumentare künftig? Diese - und viele weitere - Fragen standen am Anfang der jüngst durchgeführten Curriculumsreform, die mit dem Kursstart im Herbst 2020 in Kraft gesetzt wird.

Klar, wie der Berufsmarkt in zehn bis fünfzehn Jahren aussieht, lässt sich nur erahnen, doch die Entwicklungen in der Informationstechnologie, die gesellschaftlichen und soziodemografischen Entwicklungen (so ist u.a. ein klarer Trend hin zu einer multikulturellen Gesellschaft erkennbar) betreffen Bibliotheken direkt. Der Beruf wird insgesamt anspruchsvoller, aber auch vielfältiger. Öffentliche Bibliotheken beispielsweise entfernen sich von der stark medienzentrierten hin zu einer menschen- respektive kundenorientierten Arbeitsweise. Der Mensch steht im Mittelpunkt, nicht das Medium allein. 
So hat sich eine Arbeitsgruppe bestehend aus Dozierenden, Vertretern der Berufspraxis und des Berufsverbandes der Überarbeitung des Studienplanes angenommen mit dem Ziel, diesen an die heutigen und künftigen Erfordernisse der Berufspraxis auch im Hinblick auf die sich immer mehr durchsetzende Digitalisierung auszurichten.

\section{Aus der Praxis - für die Praxis}

Entstanden sind drei inhaltlich komplett neu gestaltete Zertifikatskurse (Certificate of Advanced Studies CAS)

\section{CAS Grundlagen der Informationspraxis}

In diesem ersten CAS sollen grundlegende Kenntnisse für die Berufsfelder Bibliothek, Archiv und Dokumentation vermittelt und die teils unterschiedlichen Vorkenntnisse der Studierenden soweit möglich ausgeglichen werden. Neben einer Einführung in die Informationswissenschaft erhalten die Studierenden die grundlegenden Branchenkenntnisse vermittelt. Sie lernen dabei, wie in Bibliotheken und Archiven gearbeitet wird. Wie und wo man Information recherchiert, ist Gegenstand des Moduls „Information Retrieval“. Und zu guter Letzt setzen sich die Studierenden mit den wichtigsten Grundregeln der modernen Führung einer Informationseinrichtung auseinander. Dabei wird ein besonderes Augenmerk auf die Auftrittskompetenz der Studierenden gelegt.

\section{CAS Bibliotheks- und Archivpraxis}

Im daran anschließenden zweiten CAS werden in einem ersten Teil Inhalte vermittelt, die wiederum für beide Fachrichtungen gleichermaßen wichtig und für die spätere Mitarbeit in einer Archiv- oder Bibliothekseinrichtung relevant sind. Dazu gehören z.B. Kenntnisse des Recordsund Informationsmanagements, der Kundenorientierung, der Öffentlichkeitsarbeit oder des Projektmanagements, die in allen IuD-Einrichtungen in den letzten Jahren zusehends an Bedeutung zugenommen haben.

\section{Wahlpflichtmodule ermöglichen persönliche Schwerpunktsetzung}

Das Studium erreicht dann im Mittelabschnitt des zweiten CAS einen ersten Höhepunkt: Mit der Wahl der Vertiefungsrichtung (Wahlpflichtmodul) setzen die Studierenden einen fachlichen Schwerpunkt und stellen damit auch die Weichen für die künftige Berufsrichtung. Bedingt durch die berufsbegleitende Tätigkeit der meisten Studierenden ist diese Richtung meist schon vorgegeben, doch kommt es hin und wieder vor, dass der Wunsch entsteht, sich in der anderen Vertiefungsrichtung zu spezialisieren.

Gegenüber dem alten, noch laufenden Curriculum erfährt der Vertiefungsteil einen massiven Ausbau von jetzt 80 auf neu 120 Lektionen. Dies entspricht fünf zusätzlichen Unterrichtstagen.

\section{Fokussierung auf öffentliche Bibliotheken}

Eine weitere signifikante Neuerung betrifft die Ausrichtung der Bibliotheksvertiefung: Das Profil wird auf den Typus der Öffentlichen Bibliotheken hin geschärft. Entsprechend sind die Inhalte auf die Bedürfnisse der Öffentlichen Bibliotheken zugeschnitten. So wird der Diskussion neuer Nutzungskonzepte viel Raum beigemessen. Die Bibliothek als sozialer Treffpunkt, als Ort der Integration und Bühne kulturellen Schaffens und als Teil der Freizeitindustrie. Einen besonderen Stellenwert wird das Thema der interkulturellen Bibliotheksarbeit einnehmen. Dass Kinder mit mehr als einer Sprache aufwachsen, ist eine Realität, der wir uns nicht verschließen dürfen. Mehrsprachigkeit sollte daher in der Bibliotheksarbeit einen festen Bestandteil einnehmen und sich entsprechend im Angebot widerspiegeln.

Abgeschlossen wird der CAS Bibliotheks- und Archivpraxis mit einer Projektwoche. Hier wird an einer praktischen Fragestellung aus der Praxis gearbeitet, die aus den Reihen der Archiv- und Bibliothekinstitutionen kommen. Dieses Konzept hat sich im aktuellen Curriculum sehr bewährt und wird auch im neuen ein integraler Bestandteil des Studienplans sein. So haben die Studierenden in den vergangenen Jahren unter anderem in einer mittelgroßen Stadtbibliothek oder einem Stiftungsarchiv eine Analyse durchgeführt und Vorschläge für deren künftige Ausrichtung gemacht. Die Projektwoche bildet so die Verknüpfung zwischen methodischem und fachlichem Wissen und deren Anwendung in der Praxis. 


\section{CAS Digitale Trends in der Informationspraxis}

Im dritten und letzten CAS steht der Erwerb von vertieftem IT-Know-how im Vordergrund. Als der technischste der drei CAS berücksichtigt er aber in der Flughöhe der Inhalte ein Publikum, das nicht gerade durch hohe Technik-Affinität besticht. Nichtsdestotrotz kommen Mitarbeitende einer Informationseinrichtung nicht mehr an vertieften Kenntnissen der Informations- und Kommunikationstechnologien vorbei. Der Arbeitsalltag in einer Informationseinrichtung ist von Informatikmitteln durchdrungen, insofern hat jemand, der sich diesen Technologien gänzlich verschließt, in unserer Branche nichts mehr zu suchen. Aber es gibt sie immer noch: Leute, die glauben, ein Bibliotheksjob bestünde primär aus Lesen...

$\mathrm{Zu}$ den neuen Inhalten gehören neben einer grundlegenden Einführung in die Informations- und Kommunikationstechnologien eine vertiefte Auseinandersetzung mit dem Umgang mit Daten aller Art. Während die einen den diskreten Umgang mit Daten fordern, wollen andere deren uneingeschränkte Verfügbarkeit: Im Studium wird beidem Rechnung getragen - Webanalytics und datenschutzrechtliche Fragestellungen sind genauso Thema wie die Anliegen der OpenGLAM-Bewegung, die vor allem Gedächtnisinstitutionen dazu anregen möchte, ihre Schätze zu teilen. Weiter kommt der Digitalisierung von analogen Medien eine größere Bedeutung zu. Die Studierenden lernen den Digitalisierungsprozess und die je nach Trägermedium unterschiedlichen technischen Anforderungen kennen und können diese Kenntnisse in unserem Labor praktisch anwenden.

Abschließend wird auch der Frage nachgegangen, inwiefern Informationsdienstleister auf die digitale Transformation reagieren. Die als Glaskugel-Modul bezeichnete Lehreinheit, soll Gelegenheit zur Reflexion und Diskussion der aktuellen Veränderungsprozesse bieten. Das beinhaltet z.B. die Auswirkungen und Potentiale von Technologien wie Virtual Reality oder der Künstlichen Intelligenz auf die Informationsdienstleister.

\section{Ständige Weiterentwicklung als Credo}

Die rasche Entwicklung der Informations- und Kommunikationstechnik ist ein wichtiger Parameter sowohl für Inhalte als auch für die methodische Gestaltung der Studienpläne für Dokumentare, Archivare und Bibliothekare in der Schweiz. Mit der Inkraftsetzung des Studienplans geht der kontinuierliche Anpassungsprozess ungehindert weiter. Es gilt auch in Zukunft, Trends zu erkennen und diese in den Studienplan einzubinden. Wenn sie auch heute und morgen noch nicht relevant für den Berufsalltag sind, so sollen sie doch in der Ausbildung frühzeitig thematisiert und die Studierenden darauf sensibilisiert werden. Damit der MAS IS auch für die nächsten 30 Jahre Bestand hat und ein Erfolgsmodell bleibt!

Links: www.fhgr.ch/mas-is; www.fhgr.ch/museum

Deskriptoren: Weiterbildung, Studium, Informationswissenschaft, Dokumentar, Archivar, Bibliothekar, Schweiz

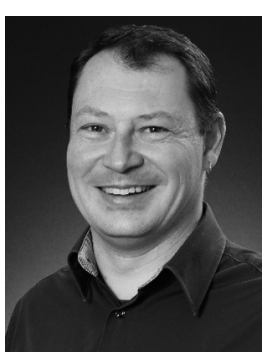

\author{
Prof. Ivo Macek \\ Fachhochschule Graubünden \\ Schweizerisches Institut für \\ Informationswissenschaft \\ Pulvermühlestrasse 57 \\ 7000 Chur \\ Schweiz \\ ivo.macek@fhgr.ch
}

Prof. Ivo Macek arbeitet seit 2005 in verschiedenen Funktionen an der Fachhochschule Graubünden. 2014 übernahm er die Leitung der Weiterbildung am Schweizerischen Institut für Informationswissenschaft SII. Er hat 2003 an der vormaligen Hochschule für Technik und Wirtschaft HTW Chur ein Fachhochschuldiplom in „Information und Dokumentation“ erworben und von 2007 bis 2009 an der DonauUniversität in Krems einen Master in „Communications and Management" absolviert. 